\title{
Tensile and compressive behaviour of S355 mild steel in a wide range of strain rates
}

\author{
Ezio Cadoni ${ }^{1}$, Daniele Forni ${ }^{1}$, Roman Gieleta ${ }^{2}$, and Leopold Kruszka ${ }^{3}$,a \\ ${ }^{1}$ DynaMat Lab, University of Applied Sciences of Southern Switzerland, Canobbio, \\ Switzerland \\ 2 Department of Mechanics and Applied Computer Science, Faculty of Mechanical \\ Engineering, Military University of Technology, 00-908 Warsaw, Poland \\ ${ }^{3}$ Faculty of Civil Engineering and Geodesy, Military University of Technology, \\ 00-908 Warsaw, Poland
}

Received 6 November 2017 / Received in final form 30 January 2018 Published online 10 September 2018

\begin{abstract}
This paper presents a comparison between mechanical compressive and tensile properties of S355 structural (mild) steel in a wide range of strain rates. A split Hopkinson pressure bar apparatus is used to investigate the dynamic compressive behaviour at high strain rates $\left(740-5880 \mathrm{~s}^{-1}\right)$. A hydro-pneumatic machine $\left(5-25 \mathrm{~s}^{-1}\right)$ and a modified split Hopkinson tensile bar apparatus $\left(300-850 \mathrm{~s}^{-1}\right)$ are used to study the strain rate sensitivity under dynamic tension. Quasi-static tensile and compressive tests are conducted on a universal electromechanical testing machine. The model parameters of two commonly used constitutive equations (Johnson-Cook and Cowper-Symonds) are also compared, separately for each model during compression and tension deformations.
\end{abstract}

\section{Introduction}

A detailed knowledge of the mechanical properties of low-carbon mild steels at different strain rates is crucial, because these steels are the most commonly used in several fields, such as, automotive, aerospace, building construction as well as off-shore structures.

During the last decades, the influence of loading rates now was the subject of several experimental studies on the mechanical behaviour of different grades of mild steels [1-4]. In particular, in the aerospace and automotive fields, the high strain-rate mechanical properties are of fundamental importance in order to improve the crashworthiness and safety against impacts. On the other hand, in building constructions and off-shore structures, a detailed knowledge of the material properties at high strain rates is essential, for example, for the assessment of robustness where a collapse may be triggered by the failure of structural elements subjected to dynamic events (e.g. earthquakes, blasts, or terrorist attacks). Among the different mild steel grades, the S355 low-alloy structural steel is widely used in the construction field due to its good strength characteristics and welding properties. The purpose of the present paper is

\footnotetext{
a e-mail: leopold.kruszka@wat.edu.pl
} 
to compare the mechanical compressive and tensile properties of the S355 structural (mild) steel in a wide range of strain rates. Hopkinson bar apparatus is employed to obtain the stress versus strain curves under dynamic loads. The material parameters of the existing Cowper-Symonds and Johnson-Cook models are determined on the basis of the mechanical properties obtained from compression tests. A comparison between these parameters and those obtained from tensile tests in a wide range of strain rates are reported as well [5].

\section{Material, samples and experimental techniques}

The object of the experimental investigations was the S355 low-alloy structural steel. The specimens for the mechanical characterisation were taken in the longitudinal direction from an hot-rolled wide-flange section HE-A. Starting from the initial section geometry, wire electrical discharge machining was used in order to obtain small prismatic samples $(7 \mathrm{~mm} \times 7 \mathrm{~mm})$. Then, the samples for the tensile and the compressive tests were obtained by turning. A cylindrical shape with the initial length $L_{0}=5 \mathrm{~mm}$ and the initial diameter $D_{0}=5 \mathrm{~mm}$ were adopted for compression tests. Consequently, the aspect ratio was $L_{0} / D_{0}=1$. Some results on the dynamic behaviour of the same material in tension have been recently published by the authors $[5,6]$. The commonly adopted geometry for dynamic testing with the split Hopkinson tensile bar (SHTB; $3 \mathrm{~mm}$ in diameter and $5 \mathrm{~mm}$ of gauge length) [5] was used for the tensile tests. In both cases the same geometry and dimensions were used for quasi-static and high strain rate tests.

Different experimental techniques were used to study the mechanical properties in tension and compression of the S355 structural steel. Quasi-static and high strain rate tests in compression were performed at the Department of Mechanics and Applied Computer Science at the Military University of Technology in Warsaw, Poland. The mechanical characterisation in tension in a wide range of strain rates was performed at DynaMat Laboratory at the University of Applied Science of Southern Switzerland.

Quasi-static compression tests were conducted at room temperature using a universal electromechanical testing machine Instron 8862 with loading speed $5 \mathrm{~mm} / \mathrm{min}$. The signals from the strain gage load cell and the dynamic extensometer with a gauge length of $12.5 \mathrm{~mm}$ were used to determine the values of nominal strains and nominal stresses and to prepare the stress-strain diagrams. Experimental compression tests at high strain rates were performed on the conventional SHPB apparatus (Fig. 1). The apparatus consists of a gas gun, incident and transmission bars, made of V720 maraging steel (BÖHLER Edelstahl GmbH \& Co KG), both $2000 \mathrm{~mm}$ long, an energy absorber, a data acquisition system, and a control system. The striker (made of V720 maraging steel) is launched using compressed gas (argon) and impacts the incident bar. This generates a compression elastic wave, which travels through the incident bar, propagates to the specimen and the transmitted bar, as well as partly reflects back in the incident bar. The details of this technique are given for example in [5]. Waves in the incident and transmitted bars are sensed by EA-06-060LZ-120 (Vishay, USA) strain gauges, which are placed in the middle of the bar. In order to measure the pressure bar signals, strain gauges connected in a quarter bridge configuration are used. The signals from the strain gauges (from the Wheatstone bridges) are conditioned with a transient amplifier LTT 500 (LTT Labortechnik Tasler GmbH, Germany) and recorded with a computer and a high-speed A/D computer board NI USB-6366 (National Instruments, USA). The main components of the SHPB apparatus are presented in Figure 1. In the described apparatus, the amplifier and the A/D computer board with $1 \mathrm{MHz}$ frequency response are used, which allows for accurately recording the transient signals from the strain gauges of the SHPB apparatus. 

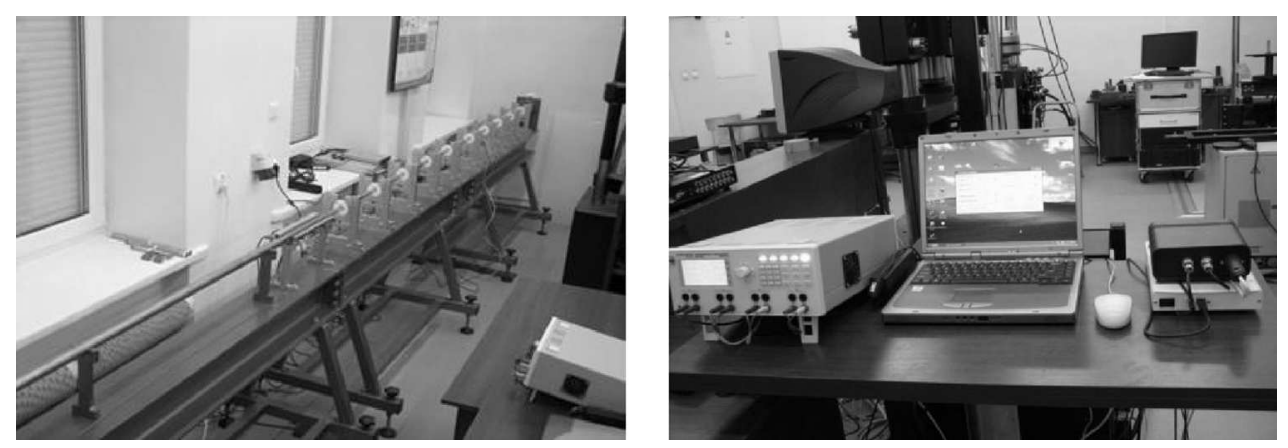

Fig. 1. General views of the Split Hopkinson Pressure Bar apparatus with data acquisition and control systems.

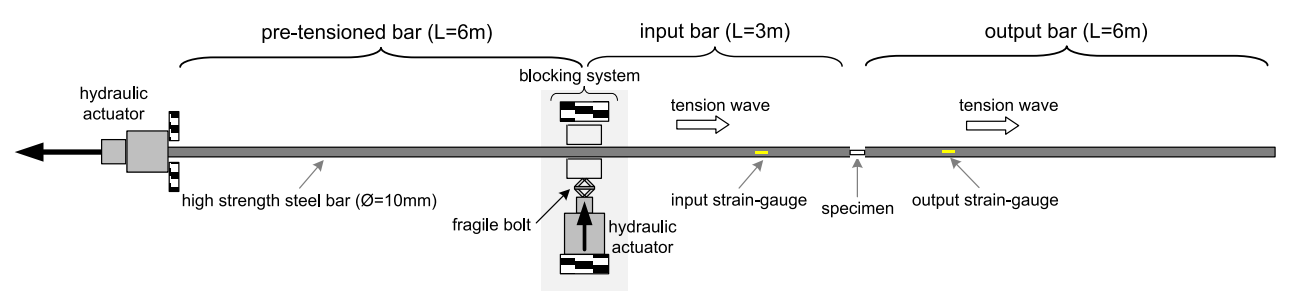

Fig. 2. JRC-split Hopkinson tensile bar set-up [5].

In the set-up, the signals are recorded digitally with a $0.5 \mu$ s sampling step. During experimental investigations, different strain rates were obtained using three striker velocities $v: 9,18$ and $27 \mathrm{~m} / \mathrm{s}$. A pulse shaping technique was used to shape the profile of the incident pulse and, as a result, to facilitate constant strain rate deformation. Disks made of copper with $D_{c}=6 \mathrm{~mm}$ and $L_{c}=1 \mathrm{~mm}$ were used as pulse shapers.

A SHTB was used for the mechanical characterisation in tension at high strain rates (Fig. 2) [7,8]. Three different testing conditions were used, leading to the following three averaged strain rates: $300 \mathrm{~s}^{-1}\left(v_{1}=2.30 \mathrm{~m} / \mathrm{s}\right), 500 \mathrm{~s}^{-1}\left(v_{2}=2.90 \mathrm{~m} / \mathrm{s}\right)$ and $850 \mathrm{~s}^{-1}\left(v_{3}=4.00 \mathrm{~m} / \mathrm{s}\right)$. The corresponding particle velocities in the input bar are reported within brackets. Tests at medium strain rates $\left(5\right.$ and $25 \mathrm{~s}^{-1}$ ) were performed by means of a hydro-pneumatic machine (HPM) described in $[8,10]$. Quasi-static tensile tests were performed by means of a universal electromechanical testing machine Zwick/Roell-Z50. All the tests were performed at room temperature.

\section{Experimental data processing}

In laboratory practice, we are often forced to cope with noised signals from different sensors. The main recommendation of experts in the signal processing field is to obtain the highest signal/noise ratio. However, it is very often difficult to completely eliminate the problem, due to the abundance of electromagnetic noise which deteriorates the quality of the measurement signals.

Nowadays, we are equipped with a new tool to denoise data from experimental tests. Filters based on the wavelets have proved to be very efficient in noise reduction. Wavelet filter functions, known as wavelets [9], decompose the signal (data) under 

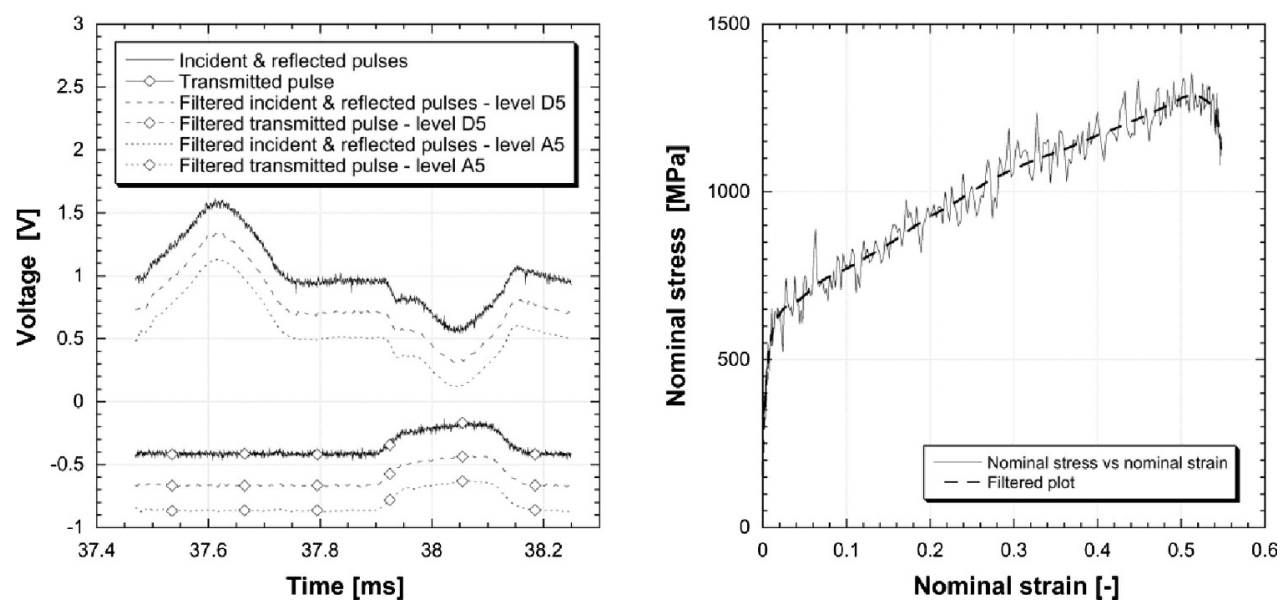

Fig. 3. Example of denoising strain gauge signals using wavelet filters (left), and example of smoothing experimental compression data (stress-strain diagram) using wavelet filter (right).

investigation into another form, was time-frequency distribution of the magnitude. The signal is decomposed into the portions of different frequencies called approximations and details, which allows easy discarding of the unwanted data in the specified signal. The theory of the wavelet transforms and wavelet filters is available for example in [11-17] and their implementation could be found in MATLAB Wavelet Toolbox and other programmes for scientists.

Experimental data from the SHPB tests were processed using Daubechies Db4 wavelet filters. Two examples of filtered recorded signals and smoothed data in the stress-strain diagram are presented in Figure 3, and they confirm the effectiveness of the wavelet filters.

In both of the split Hopkinson bars (SHPB and SHTB), the wave propagation is assumed to be governed by the one-dimensional elastic wave theory. Then, the nominal values of strains, stresses and strain rates in the sample can be calculated by the following equations [7]:

$$
\begin{aligned}
\epsilon_{N}(t) & =-2 \frac{C_{0}}{L} \int_{0}^{t} \epsilon_{r}(t) d t \\
\sigma_{N}(t) & =\frac{E A}{A_{S}} \epsilon_{t}(t) \\
\dot{\epsilon}_{N}(t) & =-2 \frac{C_{0}}{L} \epsilon_{r}(t),
\end{aligned}
$$

where $C_{0}$ is the speed of the stress wave in the pressure bar, $L$ is the length of the specimen, $E$ is the modulus of elasticity for the bar material, $A$ is the cross-sectional area of the bar, $A_{S}$ is the cross-sectional area of the specimen, $\varepsilon_{R}(t)$ is the axial strain of the reflected wave and $\varepsilon_{T}(t)$ is the axial strain of the transmitted wave. The recorded strain gauge signals from the incident and the transmitted bars are shifted in time to the specimen/bar interface. In Figure 4, typical signals from a split Hopkinson pressure bar test are shown.

In the present work, the nominal (subscript $N$ ) values of the stresses, strains and strain rates were used to determine the true (subscript $T$ ) values by the following 


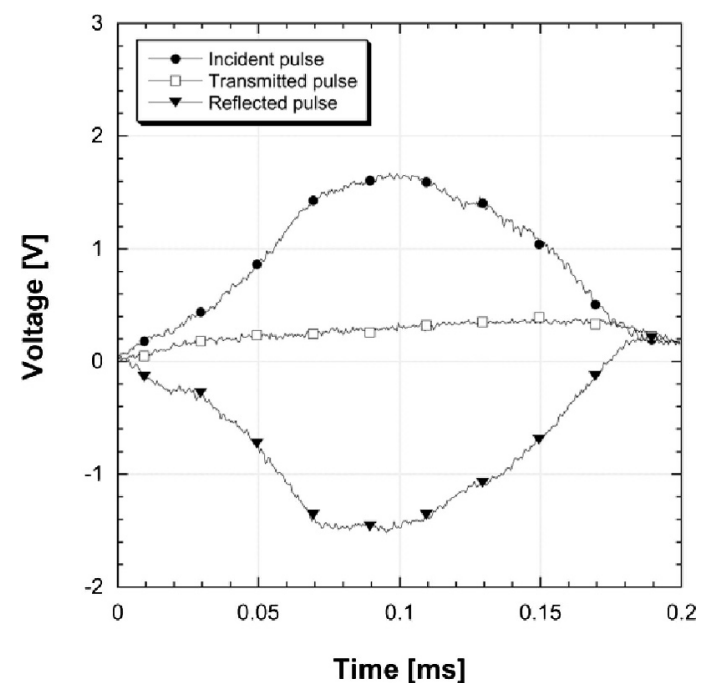

Fig. 4. Signals from strain gauges prepared for the calculation of nominal strains, stresses and strain rates $(\mathrm{SHPB})$.

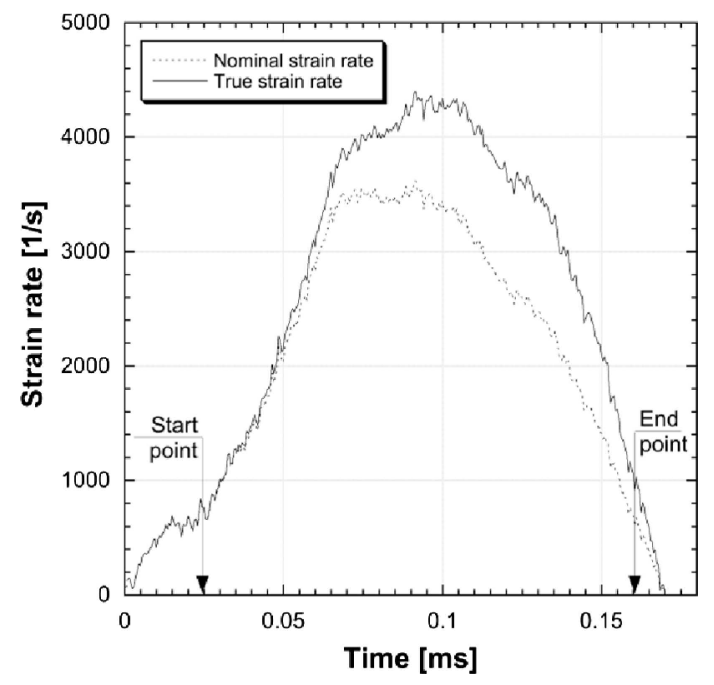

Fig. 5. Determination of the averaged true strain rate.

equations [18] (note that strains and stresses are defined as positive in compression):

$$
\begin{aligned}
& \sigma_{T}(t)=\sigma_{N}(t)\left(1-\epsilon_{N}(t)\right), \\
& \epsilon_{T}(t)=-\ln \left(1-\epsilon_{N}(t)\right), \\
& \dot{\epsilon}_{T}(t)=\frac{\dot{\epsilon}_{N}(t)}{\left(1-\epsilon_{N}(t)\right)} .
\end{aligned}
$$

The true strain rates in the compressive tests were calculated as average values from the true strain rate-time data (plots). Firstly, the yield strength for each plot was determined at $0.2 \%$ offset strain. This value, was adopted as the beginning of plastic deformation and using true stress versus time data, the start point at the 


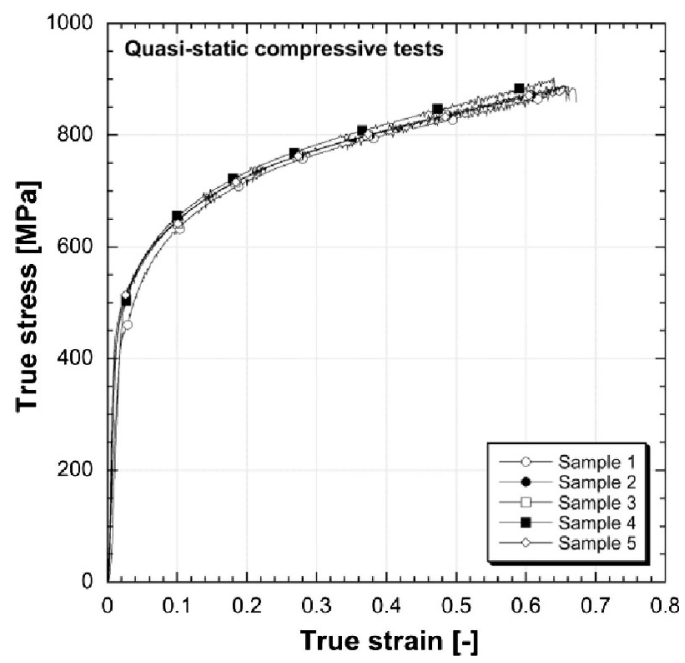

Fig. 6. True stress-strain curves for compression tests in quasi-static conditions.

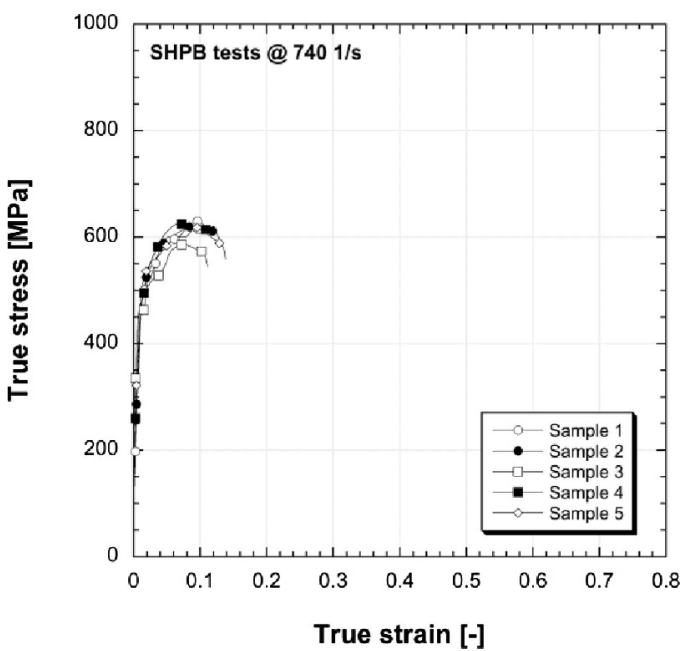

Fig. 7. True stress-strain curves for compression tests at $740 \mathrm{~s}^{-1}$.

strain rate-time plot was determined. The moment of unloading of the specimen was adopted as the end point of calculations (Fig. 5). A detailed description of the data processing and functioning of the SHTB used in this work has been reported in $[5,6]$, while the functioning of the HPM has been described in [8].

\section{Experimental results}

The results of the experimental investigations for the S355 structural steel in compression are presented in Figures 6-9 in the form of true stress-true strain diagrams. In Figures 10-15, the true curves of quasi-static and dynamic mechanical tests at the considered strain rates are depicted for tensile tests. In order to take into consideration the correction for the triaxial stress state in the tensile tests beyond the 


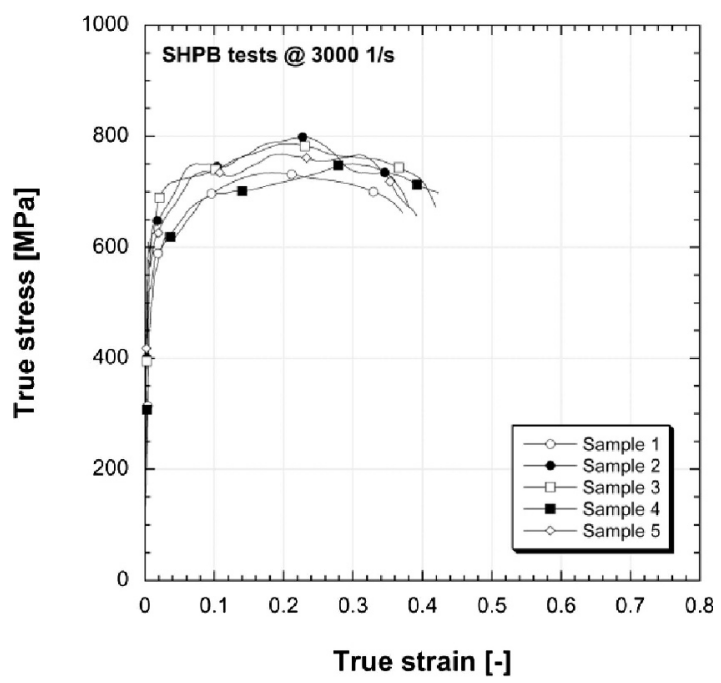

Fig. 8. True stress-strain curves for compression tests at $3000 \mathrm{~s}^{-1}$.

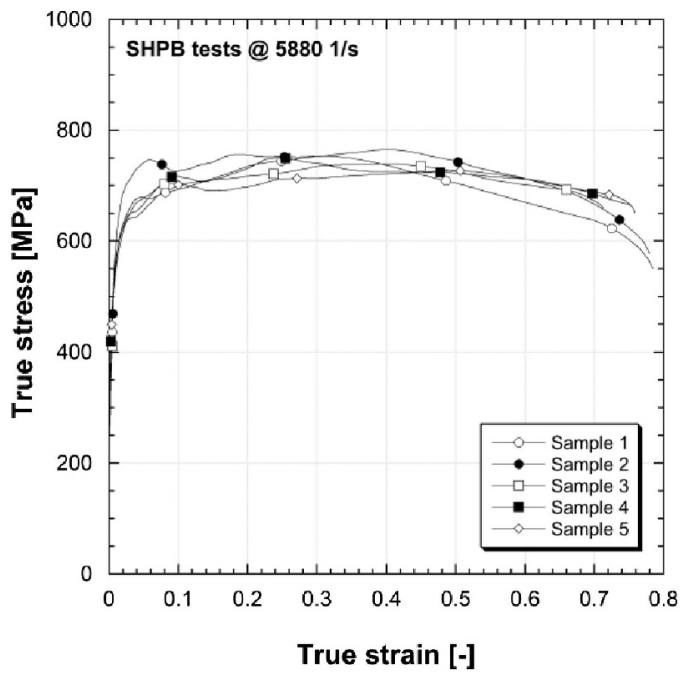

Fig. 9. True stress-strain curves for compression tests at $5880 \mathrm{~s}^{-1}$.

point of ultimate tensile strength, the one-dimensional true stress-strain curve has been reconstructed by calculating the true stress and true strain using the Bridgman formulae [19].

The strain rate sensitivity of the initial yield point for the tests both in compression and in tension is shown in Figure 16.

\section{Constitutive model development}

Different constitutive models able to characterise the flow stress are implemented in finite element codes. In the case of metals, the most popular and commonly used are the Cowper-Symonds [20] and the Johnson-Cook [21] constitutive models. 


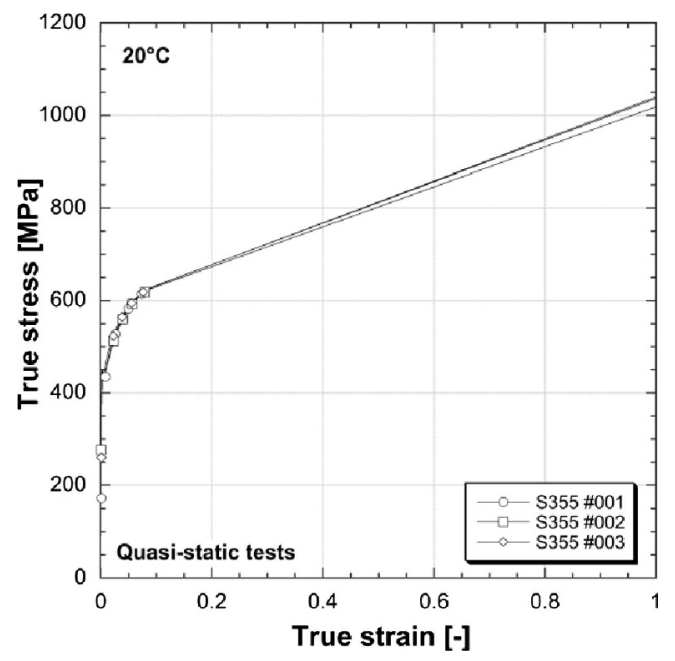

Fig. 10. True stress-strain curves for quasi-static tensile tests.

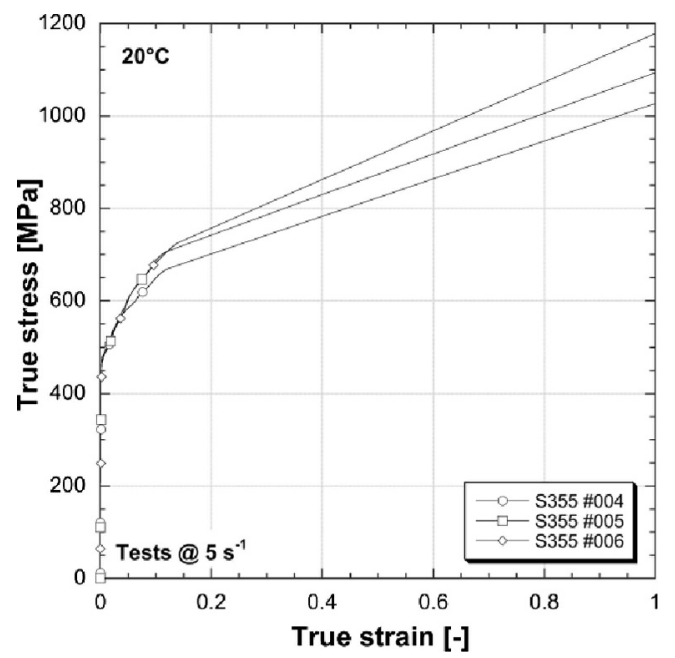

Fig. 11. True stress-strain curves for tensile tests at $5 \mathrm{~s}^{-1}$.

The Johnson-Cook and Cowper-Symonds parameters for the S355 structural steel have already been determined [5] on the basis of the mechanical behaviour in tension at room temperature (see Tab. 1). The following parameters were evaluated on the basis of the mechanical behaviour in compression. [20]:

The Cowper-Symonds model describes the flow stress by the following equation

$$
\frac{f_{y, \mathrm{dyn}}}{f_{y, \mathrm{sta}}}=1+\left(\frac{\dot{\epsilon}}{D}\right)^{1 / q}
$$

and requires only the yield strengths obtained from the quasi-static and high strain rates tests. $D$ and $q$ are constant parameters of the Cowper-Symonds model and 


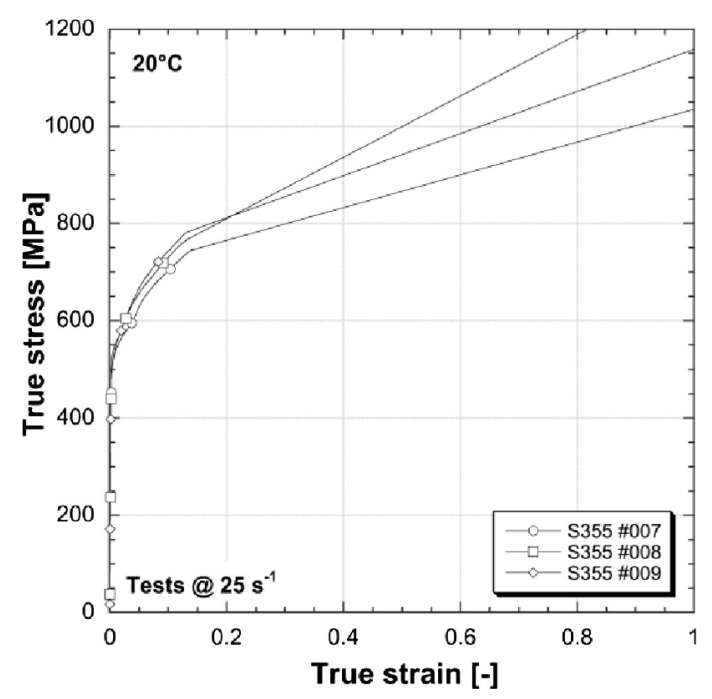

Fig. 12. True stress-strain curves for tensile tests at $25 \mathrm{~s}^{-1}$.

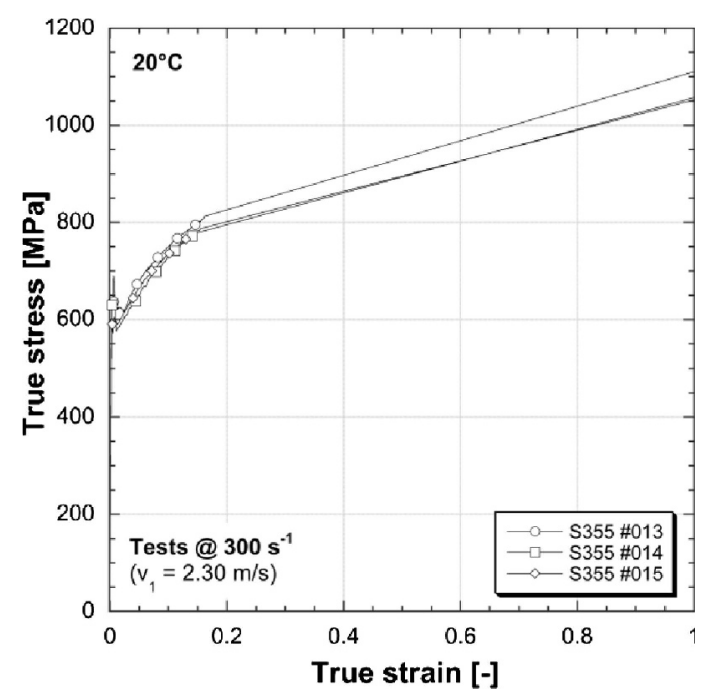

Fig. 13. True stress-strain curves for tensile tests at $300 \mathrm{~s}^{-1}$.

were obtained by means of a nonlinear square function. The result of fitting the experimental compression data to equation (7) is presented in Figure 17.

On the other hand, the flow stress of the Johnson-Cook constitutive model for our tests without taking into account the influence of the ambient temperature (i.e. at room temperature) is expressed by the following equation [21]:

$$
\sigma=\left(A+B \cdot \epsilon_{p}^{n}\right) \cdot\left(1+c \cdot \ln \frac{\dot{\epsilon}}{\dot{\epsilon_{0}}}\right)
$$

where, $A, B$ and $n$ are the parameters representing isotropic (strain) hardening, $c$ is the parameter representing strain rate hardening, $\varepsilon_{p}$ is the plastic strain, $\dot{\epsilon}$ is the considered strain rate, and $\dot{\epsilon_{0}}$ is the reference strain rate. The parameter $A$ was adopted 


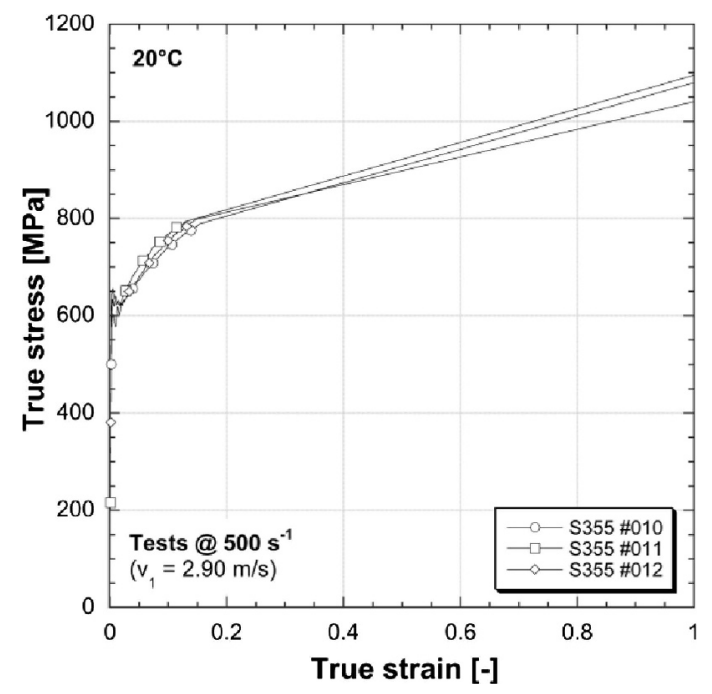

Fig. 14. True stress-strain curves for tensile tests at $500 \mathrm{~s}^{-1}$.

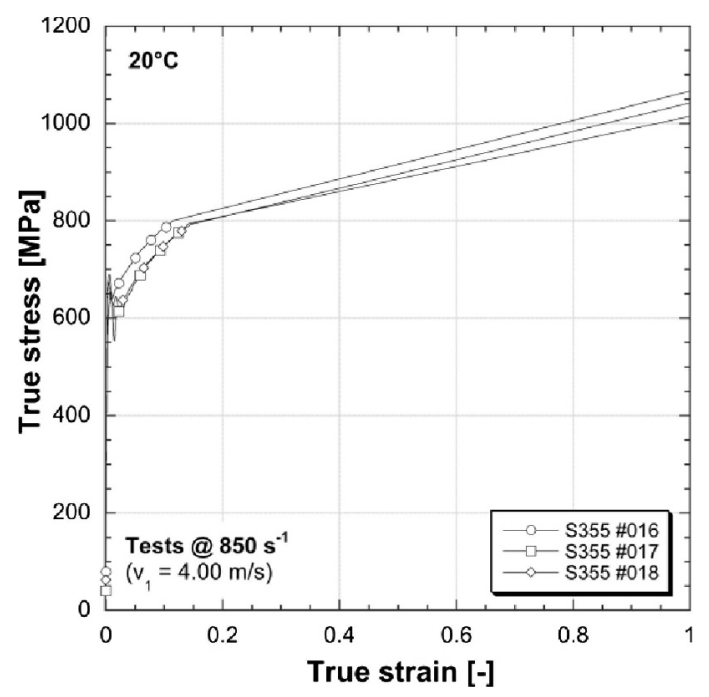

Fig. 15. True stress-strain curves for tensile tests at $850 \mathrm{~s}^{-1}$.

as the average yield strength obtained in the quasi-static tests. The parameters $B, n$ and $c$ were obtained by means of nonlinear square functions, and the results of fitting the experimental data are presented in Figures 18 and 19, respectively.

The comparison between the Johnson-Cook model and the experimental data in the dynamic compression of the S355 steel (Fig. 20) represents acceptable reliability only in the initial range of plastic strains up to $6 \%$ due to the thermal softening effect of adiabatic heating of the dynamically deformed samples. This temperature increase in the area of plastic dynamic deformation of samples' material is caused by the phenomenon of energy dissipation during passive thermomechanical couplings [22]. It is worth noting that at high strain rates no heat is dissipated from the specimen to the surrounding and the test conditions are adiabatic. As a consequence, mainly during the necking process, the temperature of the specimen rises further. This temperature 


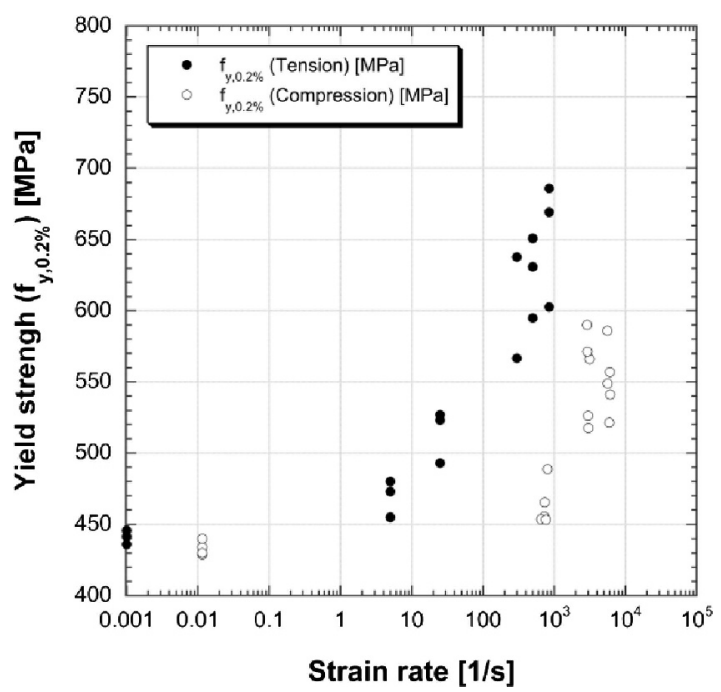

Fig. 16. Comparison between yield strength increases in tension and in compression.

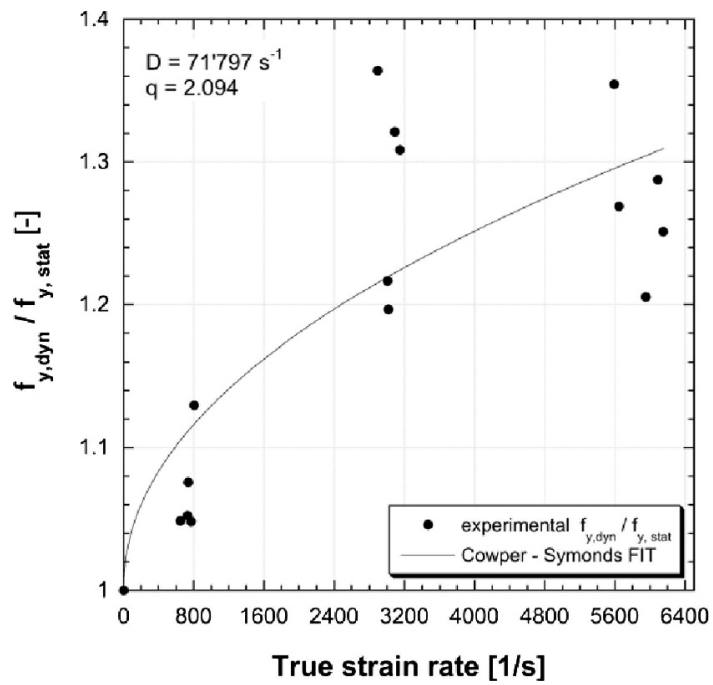

Fig. 17. Fitting experimental compression data to the Cowper-Symonds model.

increase, limited to few dozens of degrees [23,24], has been considered negligible for the purposes of the testing and hence also in the fitting method for the JohnsonCook equation the temperature increase due to adiabatic heating has been considered negligible.

The S355 steel material parameters of the existing Cowper-Symonds and Johnson-Cook models at room temperature are determined based on the experimental results as follows: Cowper-Symonds model in tension:

- Cowper-Symonds model in tension:

$$
f_{y, \text { dyn }}^{\prime}(\dot{\epsilon})=448.0 \cdot\left[1+\left(\frac{\dot{\epsilon}}{4945}\right)^{1 / 2.696}\right][\mathrm{MPa}]
$$




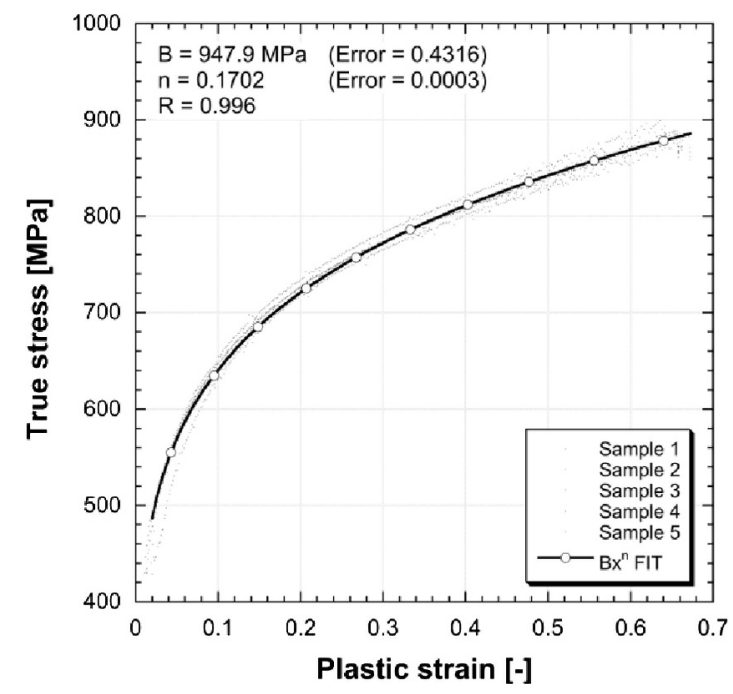

Fig. 18. Fitting experimental compression data for obtaining isotropic hardening parameters $B$ and $n$ (Johnson-Cook model).

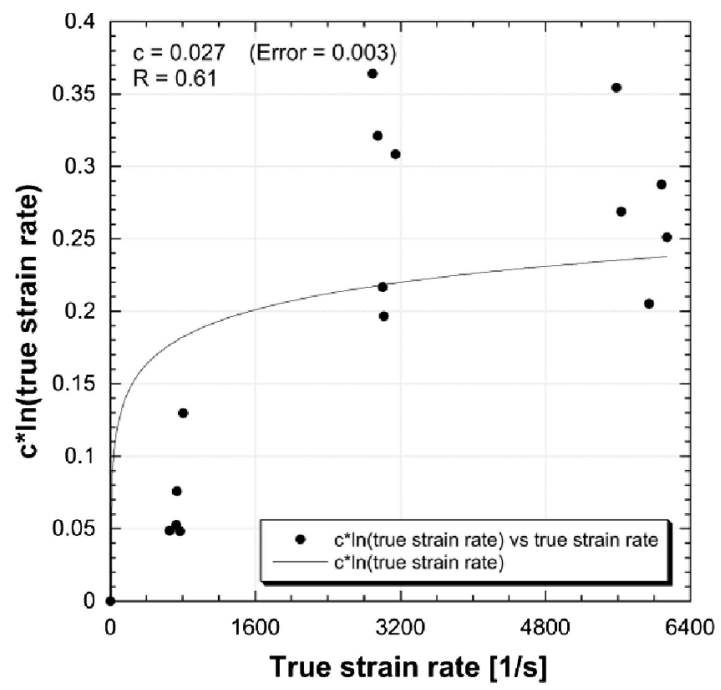

Fig. 19. Fitting experimental compression data for obtaining the strain rate sensitivity parameter $c$ (Johnson-Cook model).

- Cowper-Symonds model in compression:

$$
f_{y, \text { dyn }}^{\prime}(\dot{\epsilon})=432.6 \cdot\left[1+\left(\frac{\dot{\epsilon}}{71797}\right)^{1 / 2.094}\right][\mathrm{MPa}]
$$

- Johnson-Cook model in tension:

$$
\sigma^{t}(\epsilon, \dot{\epsilon})=\left(448.0+782.0 \cdot \epsilon_{p}^{0.5620}\right) \cdot\left(1+0.0247 \ln \frac{\dot{\epsilon}}{\dot{\epsilon}_{0}}\right)[\mathrm{MPa}]
$$




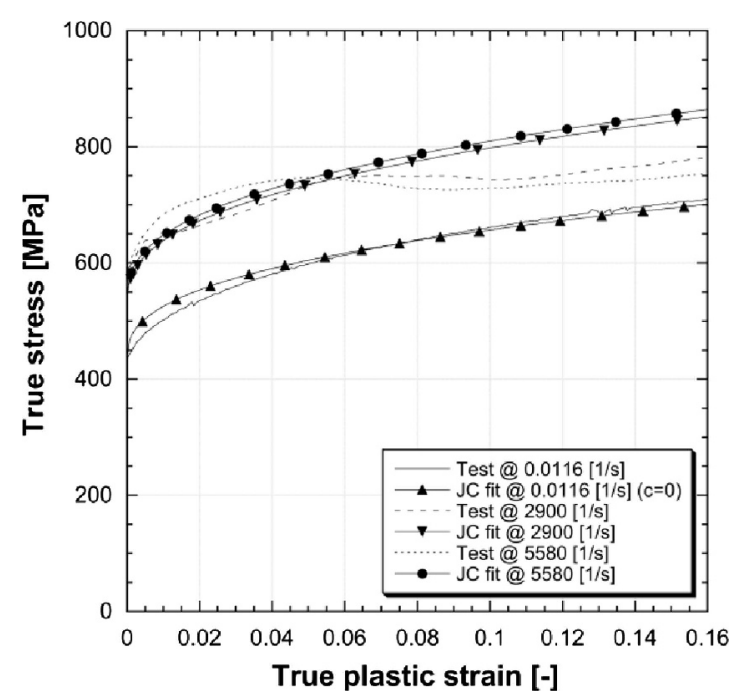

Fig. 20. Johnson-Cook model in comparison with the compressive quasi-static and SHPB results.

Table 1. Comparison between Johnson-Cook and Cowper-Symonds parameters obtained from tests in tension and compression.

\begin{tabular}{|c|c|c|c|}
\hline & & Tensile tests & Compressive tests \\
\hline \multicolumn{4}{|c|}{ Cowper-Symonds constitutive model [20] } \\
\hline$D$ & {$\left[\mathrm{~s}^{-1}\right]$} & 4945 & 71797 \\
\hline$q$ & {$[-]$} & 2.696 & 2.094 \\
\hline \multicolumn{4}{|c|}{ Johnson-Cook constitutive model [21] } \\
\hline$A$ & [MPa] & 448.0 & 432.6 \\
\hline$B$ & [MPa] & 782.0 & 947.9 \\
\hline$n$ & {$[-]$} & 0.5620 & 0.1702 \\
\hline$c$ & {$[-]$} & 0.0247 & 0.0270 \\
\hline
\end{tabular}

- Johnson-Cook model in compression:

$$
\sigma^{c}(\epsilon, \dot{\epsilon})=\left(432.6+947.9 \cdot \epsilon_{p}^{0.1702}\right) \cdot\left(1+0.0270 \ln \frac{\dot{\epsilon}}{\dot{\epsilon}_{0}}\right)[\mathrm{MPa}]
$$

The above formulas derived from the empirical modelling results of physical experiments for the same diameter samples indicate that the nature of uniaxial plastic deformation during compression and tension is not symmetrical. A significant effect on a visible difference in the described behaviours of samples made of the same material and deformed in tension and compression is due to the different boundary conditions during the tests. Thus, the asymmetry of these static and dynamic curves is a synergistic result, above all, of various boundary conditions of the samples tested during compression and tension, in particular the effect of frictional forces on the two flat surfaces of the samples, as well as various forms of real plastic deformation of tested cylindrical samples during compression (progressing barreling effect) and tension (progressing necking effect), in particular after reaching the yield stress of the material tested.

For the sake of clearness, the parameters obtained from the tensile and compressive tests are collected in Table 1. 


\section{Summary and conclusions}

The isotropic hardening and strain rate hardening of the S355 structural steel under static and dynamic tension and compression loads are discussed in this paper. The present investigations reveal that the S355 low-alloy structural steel is strain rate sensitive under both tension and compression. The comparison between the tensile and compressive properties highlights that:

- A marked increase in the tensile yield strength is noted at much lower strain rates $\left(10^{1} \mathrm{~s}^{-1}\right)$ than for the compressive yield strength $\left(10^{3} \mathrm{~s}^{-1}\right)$ - see Figure 16.

- The yield strength is generally higher in the tensile tests. At $10^{3} \mathrm{~s}^{-1}$, the tensile yield strength is higher $(40 \%)$ than the highest compressive yield strength - see Figure 16.

- The parameters for the existing Cowper-Symonds and Johnson-Cook models are determined. The flow stress obtained from these constitutive models with estimated material parameters are in good agreement with the experimental results. In the comparison of the compression and tension empirical models, a non-symmetrical behaviour is observed.

Similar results for this material in terms of strain rate sensitivity ( $C$ term of the Johnson-Cook constitutive equation), were also reported in [3,4]. They found $C=0.039$ evaluated for tests at $600 \mathrm{~s}^{-1}$. The $C$ parameter reported in this research $(C=0.0247)$ has been evaluated as a fitted value for tests from 5 to $850 \mathrm{~s}^{-1}$. By the way, the $C$ parameter extrapolated for similar conditions, e.g. only for tests at $500 \mathrm{~s}^{-1}(C=0.0318)$, is more similar to that reported in $[3,4]$. Some of the tensile data reported in this work are also part of two previously published papers [5,6]. The novelty of the current work is the publication the whole data in terms of true stress true strain curves. This has allowed a better graphical comparison between the compressive and tensile tests. Moreover, from these graphs (see Figs. 6-15) it is possible to observe the excellent repeatability of the performed tests.

The authors wish to thank M. Dotta and N. Tesio of the DynaMat Laboratory, University of Applied Sciences of Southern Switzerland, for their assistance in the experiments.

\section{Author contribution statement}

The experimental analysis of the S355 steel behavior during static and dynamic tension including empirical constitutive modeling was carried out by Swiss authors. On the other hand, such same analysis concerning the study of this steel, but during compression conditions, was performed by the Polish authors.

Open Access This is an open access article distributed under the terms of the Creative Commons Attribution License (http://creativecommons.org/licenses/by/4.0), which permits unrestricted use, distribution, and reproduction in any medium, provided the original work is properly cited.

\section{References}

1. N.K. Singh, E. Cadoni, M.K. Singha, N.K. Gupta, J. Eng. Mech. 139, 1197 (2013)

2. M. Singh, D. Sood, R.K. Gupta, P C. Gautam, B. Sewak, A.C. Sharma, M. Thomson, Def. Sci. J. 58, 275 (2008) 
3. J. Ribeiro, A. Santiago, C. Rigueiro, in Proceedings of the XIV Portuguese Conference on Fracture, PCF2014, edited by A. de Jesus, A. Ribeiro, J. Morais, J. Xavier, N. Dourado (University of Trás-os-Montes e Alto Douro, 2014)

4. J. Ribeiro, A. Santiago, C. Rigueiro, IJSI 7, 323 (2016)

5. D. Forni, B. Chiaia, E. Cadoni, Eng. Struct. 119, 164 (2016)

6. D. Forni, B. Chiaia, E. Cadoni, in Proceedings of the VII European Congress on Computational Methods in Applied Sciences and Engineering, ECCOMAS2016, edited by M. Papadrakakis, V. Papadopoulos, G. Stefanou, V. Plevris (Institute of Structural Analysis and Antiseismic Research, Greece, 2016), p. 4920

7. W. Chen, B. Song, Split Hopkinson (Kolsky) bar: design, testing and applications (Springer, Berlin, 2011)

8. E. Cadoni, M. Dotta, D. Forni, S. Bianchi, Appl. Mech. Mater. 82, 124 (2011)

9. E. Cadoni, M. Dotta, D. Forni, N. Tesio, Appl. Mech. Mater. 82, 86 (2011)

10. E. Cadoni, M. Dotta, D. Forni, P. Spatig, J. Nucl. Mater. 414, 360 (2011)

11. P. Addison, The illustrated wavelet transform handbook (Taylor \& Francis, New York, 2002)

12. C.S. Burrus, R.A. Gopinath, H. Guo, Introduction to wavelets and wavelet transforms: a prime (Prentice Hall, New Jersey, 1998)

13. I. Daubechies, Ten lectures on wavelets (Society for Industrial and Applied Mathematics, Philadelphia, Pennsylvania, 1992)

14. S. Mallat, A wavelet tour of signal processing: the sparse way (Academic Press, Elsevier, 2009)

15. J.S. Walker, A primer on wavelets and their scientific applications (Chapman \& Hall/CRC, Taylor \& Francis Group, 2008)

16. D.L. Fugal, Conceptual wavelets in digital signal processing: an in-depth practical approach for the non-mathematician (Space and Signals Technical Publishing, San Diego, California, 2009)

17. G. Strang, T. Nguyen, Wavelets and filter banks (Wellesley-Cambridge Press, Wellesley, USA, 1997)

18. W.N. Sharpe, Springer handbook of experimental solid mechanics (Springer, Berlin, 2008)

19. P. Bridgman, Studies in large plastic flow and fracture (McGraw-Hill, New York, 1952)

20. G.J. Cowper, P.S. Symonds, Report, Brown University, Division of Applied Mathematics, 1957

21. G.R. Johnson, W.H. Cook, in Proceedings of the Seventh International Symposium on Ballistics, The Hague, 1983 (American Defense Preparedness Association, Koninklijk Instituut van Ingenieurs, Netherlands, 1983), p. 541

22. L. Kruszka, W.K. Nowacki, J. Therm. Stress. 18, 313 (1995)

23. R. Kapoor, S. Nemat-Nasser, Mech. Mater. 27, 1 (1998)

24. J.P. Castellanos, A. Rusinek, J. Theor. Appl. Mech. 50, 337 (2012) 